\title{
ATIVAÇÃO E CO-CONTRAÇÃO DOS \\ MÚSCULOS GASTROCNÊMIO E TIBIAL \\ ANTERIOR NA MARCHA DE MULHERES \\ UTILIZANDO DIFERENTES ALTURAS DE SALTOS
}

\author{
DRA. CLÁUDIA TARRAGÔ CANDOTTI \\ Doutora em Ciências do Movimento Humano pelo Programa de Pós-Graduação \\ em Ciências do Movimento Humano (ESEF/UFRGS) \\ Professora do Curso de Fisioterapia e do Programa de Pós-Graduação (Mestrado e Doutorado) \\ da Universidade Federal do Rio Grande do Sul (Porto Alegre - Rio Grande do Sul - Brasil) \\ E-mail: claudia.candotti@ufrgs.br
}

GRAD. KÁTYA VIEIRA DE CARVALHO

Graduada em Educação Física pela Universidade do Vale do Rio dos Sinos (UNISINOS)

(São Leopoldo - Rio Grande do Sul - Brasil)

E-mail: katyacarvalho@gmail.com

\section{MS. MARCELO LA TORRE}

Doutorando em Ciências do Movimento Humano pelo Programa de Pós-Graduação

em Ciências do Movimento Humano (ESEF/UFRGS)

Professor do Curso de Fisioterapia e Educação Física da Universidade do Vale do Rio dos Sinos (São Leopoldo - Rio Grande do Sul - Brasil)

E-mail:marcelotorre@unisinos.br

\section{GRAD. MATIAS NOLL}

Mestrando em Ciências do Movimento Humano pelo Programa de Pós-Graduação em Ciências do Movimento Humano (ESEF/NFRGS) (Porto Alegre - Rio Grande do Sul - Brasil)

E-mail: matiasnoll@yahoo.com.br

\section{GRAD. MARCELO VARELA}

Graduado em Fisioterapia pela Universidade do Vale do Rio dos Sinos (UNISINOS)

(São Leopoldo - Rio Grande do Sul - Brasil)

E-mail: marcelogvarela@bol.com.br 


\section{RESUMO}

O objetivo deste estudo foi verificar a ativação e a co-contração muscular dos músculos tibial anterior (TA) e gastrocnêmio lateral (GL) durante a marcha, utilizando diferentes calçados. Nove mulheres caminharam sobre uma esteira elétrica com velocidade de 3,5 $\mathrm{km} / \mathrm{h}$ em três situações: sem calçado, com salto baixo $(6 \mathrm{~cm})$ e com salto alto $(9 \mathrm{~cm})$, sendo simultaneamente registrados sinais eletromiográficos do TA e GL. Os principais resultados encontrados no presente estudo demonstraram que: ( I ) independente da altura do salto houve maior ativação do GL ( $p<0,05)$, em comparação com o TA; (2) o músculo GL apresentou maior ativação $(p<0,05)$ com o calçado de salto alto, em comparação a situação com os pés descalços; e (3) quanto maior a altura do salto, maiores percentuais de co-contração muscular foram encontrados $(p<0,05)$.

PALAVRAS-CHAVE: Marcha; eletromiografia; co-contração; avaliação.

\section{INTRODUÇÃO}

As pesquisas da área de Biomecânica têm dedicado grande parcela do seu interesse no estudo da marcha (CANDOTTI; LOSS, 2006), seja do ponto de vista cinético (CAMPOS et al, 2002), cinemático (HAMILL; KNUTZEN, I999; MCGINNIS, 2002) ou neuromuscular (SANTOS et al., 2008; CORREA et al., 2002; FONSECA et al., 200 I). Alguns destes estudos têm sido desenvolvidos com intuito de avaliar a funcionalidade da marcha com a utilização de diferentes tipos de calçados e com diferentes alturas de saltos.

Dentre os inúmeros estudos que objetivam avaliar os aspectos neuromusculares da marcha humana por meio da eletromiografia de superfície (EMG), poucos são os estudos que investigaram o comportamento neuromuscular do músculo tibial anterior e gastrocnêmio lateral, bem como o nível de co-contração entre estes músculos, tanto na marcha normal quanto na marcha com utilização de calçado de salto (IUNES; SANTOS, 2005; CORRÊA et al., 2002). A co-contração pode ser definida como a realização de uma ativação neuromuscular simultânea de dois ou mais músculos para gerar uma estabilização ou ajuste dinâmico da rigidez das partes móveis, permitindo movimentos e ajustes posturais (FONSECA et al., 200 I ; NUNES, 2004), sendo também caracterizada como o controle da estabilidade mais eficiente para o ajuste dinâmico (AQUINO et al., 2004). Embora existam muitos estudos desta natureza, os quais objetivam avaliar o comportamento neuromuscular dos músculos envolvidos na marcha (SANTOS et al., 2008; CHANG et al., 2001 ; EISENHARDT et al., 1996; SNOW; WILLIANS, I994), foram encontrados poucos voltados para a marcha com utilização de salto alto, fato este que motivou a realização do presente estudo. 
A utilização de sapatos de salto alto altera o padrão postural e neuromuscular da marcha, visto que coloca o corpo sob condições não fisiológicas, alterando a função de articulações, e gerando nelas forças e torques acima do normalmente exercido. A articulação do joelho, por exemplo, é submetida, com o uso de sapatos de saltos, a torques excessivos, sendo exposta às transformações degenerativas da cartilagem articular e à ocorrência de osteoartrite (KERRIGAN; TODD; RILEY, 1998). Não obstante, tem sido também relatado que o uso de salto alto acarreta uma sobrecarga no suporte anterior do pé, estando relacionada com a ocorrência de dor na região metatarsiana (KENDALL; MCCREARY; PROVANCE, 1995).

O uso freqüente do salto alto provoca uma redução da função do calcanhar na sustentação do corpo, acarretando desequilíbrio da musculatura agonista e antagonista (IUNES; SANTOS, 2005; SILVA, 2007), visto que o salto alto obriga o usuário a adotar uma posição prolongada de flexão plantar (JAHSS, 1982). Isto pode resultar em encurtamento dos músculos gastrocnêmios e sóleo, fato que pode restringir a flexão dorsal do tornozelo e ocasionar a hiperextensão do joelho na posição em pé (JAHSS, 1982), e resultar em desconforto às pessoas já habituadas ao uso do salto no momento em que forem utilizar um calçado de sola plana (IUNES et al., 2008).

Assim, considerando que ambos os músculos, tibial anterior e gastrocnêmio, possuem uma grande relevância funcional no ato de caminhar, entende-se importante desenvolver estudos que busquem compreender o padrão de ativação e de co-contração destes músculos, tanto na marcha normal quanto na marcha com utilização de calçado de salto. Deste modo, o presente estudo tem como objetivo verificar a ativação e co-contração neuromuscular dos músculos gastrocnêmio lateral e tibial anterior durante a marcha de mulheres usando diferentes calçados, com variadas alturas de saltos. Especula-se que, independente da altura do salto, ocorre maior ativação do gastrocnêmio lateral em comparação com o tibial anterior, e que, quanto mais alto for o salto, maior será o percentual de co-contração muscular entre o gastrocnêmio lateral e o tibial anterior.

\section{MÉTODO}

\section{AMOSTRA}

A amostra foi composta por nove mulheres universitárias, com média de idade, estatura e massa corporal de 22,3 $\pm 3,3$ anos, $163 \pm 10 \mathrm{~cm}$ e 53,3 $\pm 4,1$ $\mathrm{Kg}$, respectivamente.

O critério de inclusão foi utilizar salto alto com freqüência mínima de uma vez por semana, sendo que a freqüência média do uso semanal de salto alto foi de 
2,4 \pm 0,7 dias/sem. Os critérios de exclusão foram possuir diagnóstico clínico de qualquer patologia na coluna vertebral e nos membros inferiores, bem como referir dor durante a caminhada. O estudo respeitou a Resolução 196/96 do Conselho Nacional de Saúde, sendo garantido o sigilo dos dados pessoais e confidenciais. Além disso, foram fornecidas, antes dos testes, informações detalhadas a respeito dos procedimentos do estudo. Todas as mulheres participaram voluntariamente da pesquisa e assinaram o Termo de Consentimento Livre e Informado.

A avaliação da marcha foi realizada no Laboratório de Fisiologia do Exercício e Cineantropometria da Instituição de origem dos pesquisadores. As participantes foram avaliadas em um único dia, pelos mesmos avaliadores.

Inicialmente foi solicitado que cada indivíduo caminhasse, antes de iniciar o protocolo de avaliação, durante cinco minutos, sobre a esteira ergométrica Inbramed (Inbrasport, Porto Alegre, Brasil), para que houvesse uma familiarização com o equipamento e aquecimento muscular. Durante este aquecimento, os indivíduos caminharam sem calçado, com velocidade livre, desde que não ultrapassasse a velocidade do protocolo de avaliação $(3,5 \mathrm{Km} / \mathrm{h})$.

Em seguida, foram realizados os testes de Contração Voluntária Máxima (CVM) dos músculos gastrocnêmio lateral e tibial anterior. Para a realização destes testes, os indivíduos permaneceram sentados no solo, com quadril flexionado em $90^{\circ}$ e joelhos estendidos, tendo as extremidades inferiores e a região dorsal imobilizadas (KONRAD, 2005). Para avaliação da CVM do gastrocnêmio lateral foi solicitado que os indivíduos realizassem a máxima flexão plantar. Após dois minutos de repouso, os indivíduos realizaram a máxima flexão dorsal do tornozelo para avaliar a CVM do tibial anterior (KONRAD, 2005). Este teste de CVM foi repetido por três tentativas, para ambos os músculos, sendo simultaneamente monitorada a atividade eletromiográfica.

\section{PROTOCOLO DE AVALIAÇÃO}

O protocolo de avaliação consistiu em caminhar sobre uma esteira ergométrica em três diferentes situações: sem calçado, com salto baixo $(6 \mathrm{~cm}$ de altura) e com salto alto ( $9 \mathrm{~cm}$ de altura) (Figura I). Os testes foram realizados de forma aleatória, para evitar que a ordem de execução pudesse interferir nos resultados. Os calçados foram disponibilizados pelos pesquisadores. A velocidade da marcha foi de $3,5 \mathrm{~km} / \mathrm{h}$, e o tempo de realização da marcha foi de dois minutos. Entre os três modos de caminhada, deu-se um intervalo de cinco minutos. 

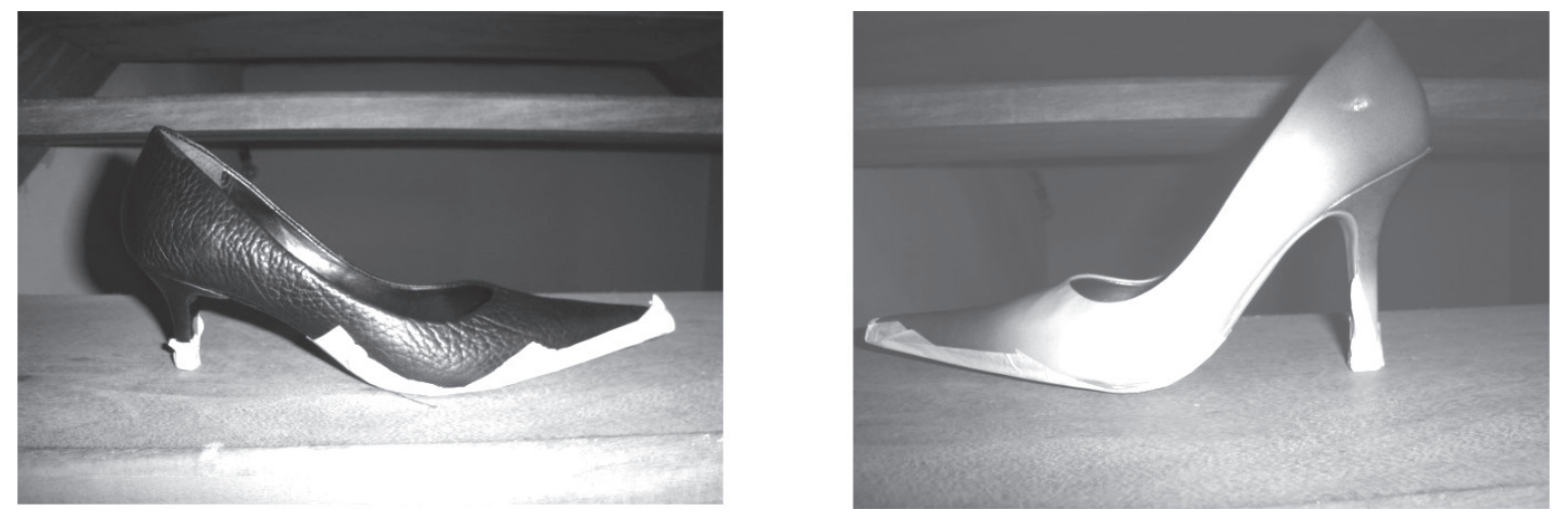

Figura I. Ilustração dos calçados utilizados na avaliação: (a) salto baixo e (b) salto alto.

\section{PROCEDIMENTOS DE AQUISIÇÃO DOS SINAIS}

Inicialmente foram realizados os procedimentos adequados para o registro do sinal EMG, como a depilação e limpeza da pele com álcool, colocação dos eletrodos e verificação da impedância (aceita quando inferior a $5 \mathrm{~K} \Omega$ ). Foram utilizados eletrodos de superfície descartáveis (Ag/AgCl; com diâmetro de 2,2cm; com adesivo de fixação), em configuração bipolar, dispostos longitudinalmente às fibras musculares, sendo, dois pares de eletrodos posicionados sobre os músculos gastrocnêmio lateral (GL) e tibial anterior (TA), nos membros inferiores direito e esquerdo. Os eletrodos no tibial anterior foram posicionados no terço proximal da linha traçada entre a fíbula e o maléolo medial. Já no gastrocnêmio lateral, foram posicionados no terço proximal da linha entre a cabeça da fíbula e o centro do calcanhar (SENIAM, 2008). O eletrodo de referência foi posicionado na crista-ilíaca.

Para a aquisição dos dados eletromiográficos foi utilizado um eletromiógrafo de quatro canais (Miotec Equipamentos Biomédicos, Porto Alegre, Brasil) conectado a um computador (SONY 2G DDR/RAM - 500 GB). A recolha dos sinais de eletromiografia foi realizada com o software Miograph 2.0 (Miotec Equipamentos Biomédicos, Porto Alegre, Brasil). A freqüência de amostragem foi de $1000 \mathrm{~Hz}$ para cada músculo.

Durante a realização do protocolo, foi realizada a filmagem da marcha utilizando uma webcam integrada ao sistema de aquisição de dados (Miotec Equipamentos Biomédicos, Porto Alegre, Brasil), com uma freqüência de amostragem de $15 \mathrm{~Hz}$. A filmagem foi necessária para obtenção do momento temporal do heel strike (contato do calcanhar) e do toe off (retirada da ponta do pé), importantes para determinação do ciclo da marcha. 
A análise e o processamento do sinal EMG foram realizados no software SAD (www.ufgrs.br/lmm). Inicialmente foi realizado o procedimento de filtragem digital utilizando os filtros Passa-Alta $(\mathrm{IOHz})$ e Butterworth $(20-500 \mathrm{~Hz})$. Após esta fase, o sinal EMG foi processado no domínio do tempo, a partir do valor do envelope Root Mean Square (RMS), com janelamento móvel de 0,5 segundos, do tipo Hamming. Os sinais foram recortados em função da fase de apoio do ciclo da marcha, a partir do conhecimento do momento temporal de heel strike e toe off. Para normalização da amplitude do sinal EMG, foi utilizado como critério o valor máximo atingido no teste de CVM. Para análise, foram consideradas apenas dez fases de apoio do ciclo da marcha, retirados depois de transcorrido um minuto de caminhada, correspondente ao tempo em que os indivíduos caminharam sobre a esteira em cada situação de teste: pés descalços, salto baixo e salto alto. Utilizando os dez ciclos, foi calculada a média aritmética para fornecer o nível de ativação de cada situação de teste.

Além disto, foi calculado o valor da integral do sinal EMG (Trapezius) de cada um dos músculos, a fim de obter o percentual de co-contração entre os músculos. O percentual de co-contração entre o gastrocnêmio lateral e tibial anterior foi calculado de acordo com a Equação I (WINTER, 2005).

Equação I

$$
\% \text { COCON }=2 \times \frac{\text { areacomum } A \& B}{\text { area } A+\text { area } B} \times 100 \%
$$

onde:

\% COCON = percentual de co-contração entre os dois músculos antagonistas. area $A$ = área abaixo do sinal EMG envelopado da curva do músculo $A$. area $B=$ área abaixo do sinal EMG envelopado da curva do músculo $B$. areacomum $A \& B=$ área comum de atividade entre dois músculos antagonistas.

\section{TRATAMENTO ESTATÍSTICO}

A análise estatística foi realizada no software SPSS 17.0, utilizando a média de ativação de cada músculo e o valor do percentual de co-contração em cada uma das três situações de teste. Confirmada a normalidade e homogeneidade dos dados, a partir do teste Shapiro-Wilk, os mesmos foram submetidos a Anova One-Way e o post hoc de Bonferroni, para verificar as diferenças entre as três situações de 
teste (I) da ativação neuromuscular dos músculos TA e GL e (2) do percentual de co-contração. O nível de significância foi de 0,05.

\section{RESULTADOS}

A média de ativação apresentada pelos músculos tibial anterior e gastrocnêmio lateral, nas diferentes situações de teste (pés descalços, com salto baixo e com salto alto), é apresentada na Figura 2. Os resultados da Anova One-Way indicaram que existe diferença significativa entre o nível de ativação do tibial anterior e gastrocnêmio lateral, tanto para o lado direito $(p=0,00 \mathrm{I})$, como para o lado esquerdo $(p=0,00 \mathrm{I}$ ), nas três situações de teste (Figura 2$)$. Estes resultados demonstram que, independente da situação do teste (pés descalços, com salto baixo e com salto alto), ocorreu maior ativação do gastrocnêmio lateral, em comparação com o tibial anterior.

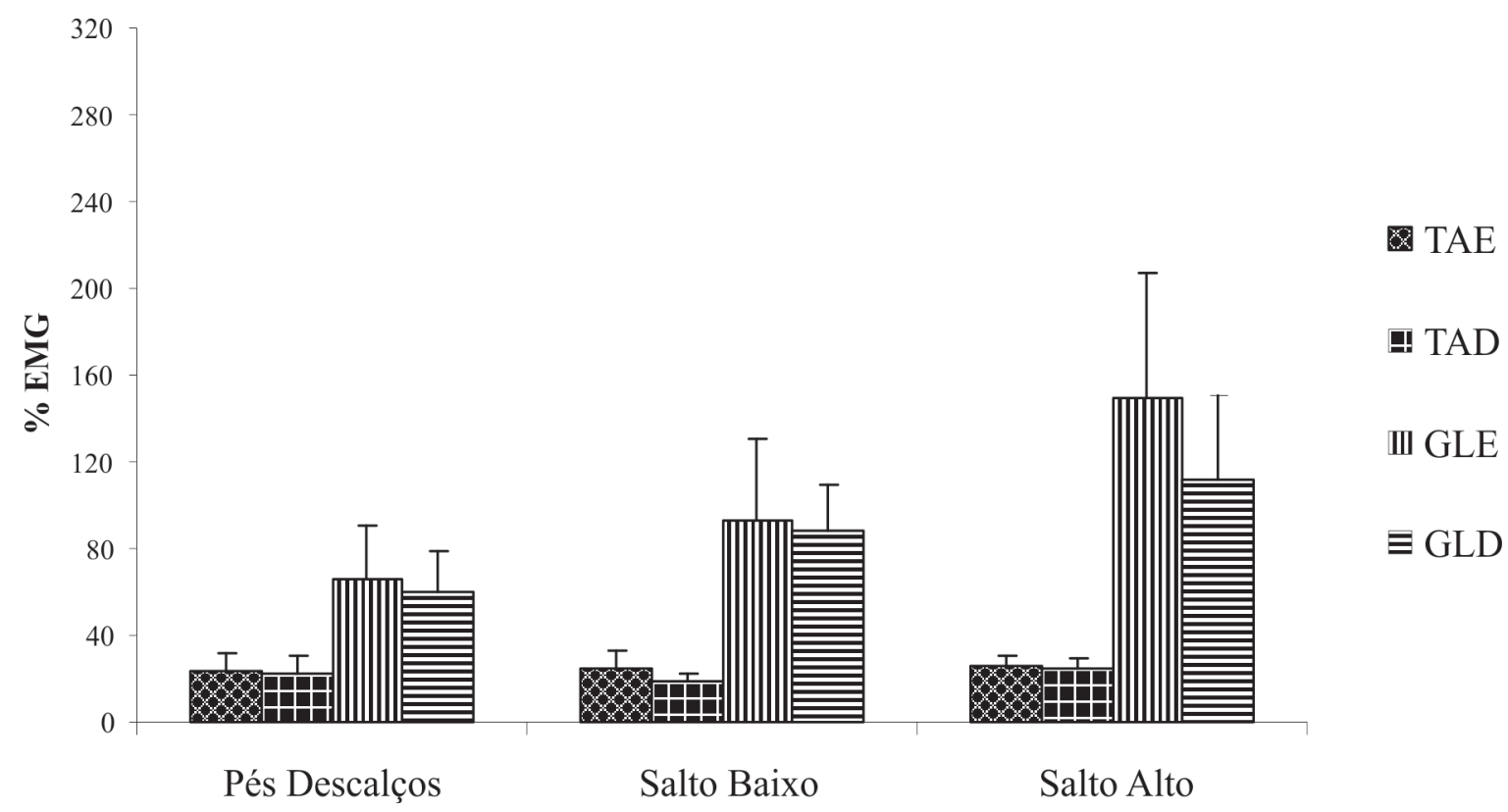

Figura 2. Média e desvio padrão do nível de ativação neuromuscular dos músculos tibial anterior direito (TAD), tibial anterior esquerdo (TAE), gastrocnêmio lateral direito (GLD) e gastrocnêmico lateral esquerdo (GLE) nas diferentes situações de teste: pés descalços, salto baixo e salto alto. * Diferença significativa $(p<0,05)$.

A Figura 2 apresenta o resultado da comparação entre as três situações de teste. Pode-se observar que não houve diferença significativa para a ativação dos músculos tibial anterior direito $(p=0,248)$ e tibial anterior esquerdo $(p=0,774)$ entre as três situações de teste. Entretanto, para o músculo gastrocnêmio lateral, a comparação entre as três situações de teste demonstrou que houve diferença apenas entre as situações "pés descalços $\times$ salto alto", tanto para o lado direito $(p=0,020)$ quanto para o lado esquerdo $(p=0,039)$, sendo maior a ativação com o salto alto, independente do lado (Figura 2). 


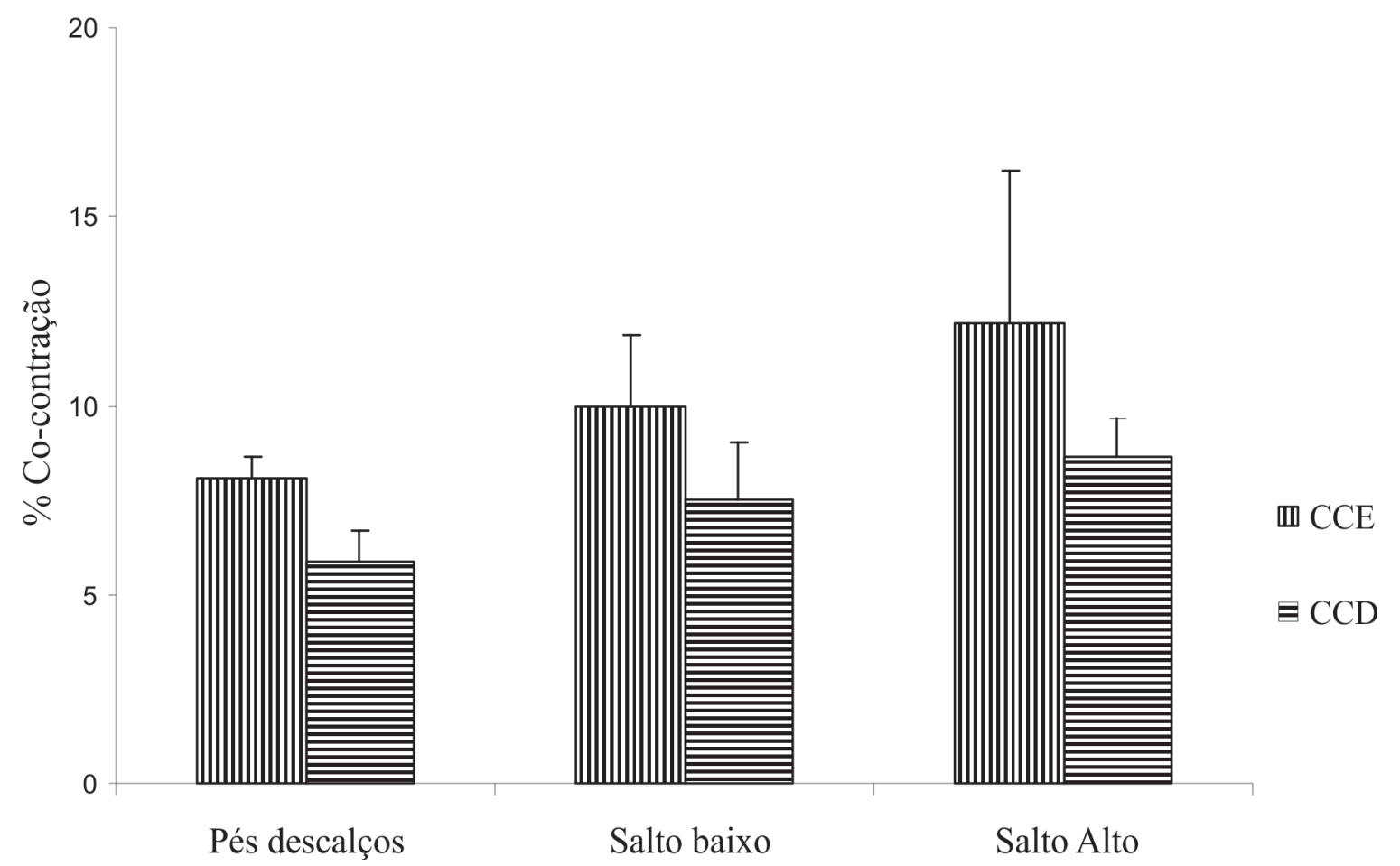

Figura 3. Média e desvio padrão do percentual de co-contração entre os músculos tibial anterior e gastrocnêmio lateral nos membros inferiores, esquerdo (CCE) e direito (CCD), nas diferentes situações de teste: pés descalços, salto baixo e salto alto. * Diferença significativa $(p<0,05)$.

A Figura 3 apresenta o percentual de co-contração entre os músculos gastrocnêmio lateral e tibial anterior dos membros inferiores, lados direito e esquerdo separadamente, nas três situações de teste. Os resultados demonstraram que as diferenças do percentual de co-contração no lado direito foram entre as situações "pés descalços $\times$ salto baixo" ( $p=0,043)$ e "pés descalços $\times$ salto alto" ( $p=0,00$ I). Já no lado esquerdo, as diferenças de co-contração ocorreram apenas entre as situações "pés descalços $x$ salto alto" ( $p=0,035)$ (Figura 3). Estes resultados demonstram que, quanto maior a altura do salto, maiores percentuais de co-contração muscular foram encontrados.

\section{DISCUSSÃO}

O objetivo deste estudo foi verificar a ativação e co-contração neuromuscular dos músculos gastrocnêmio lateral (GL) e tibial anterior (TA) durante a marcha de mulheres usando diferentes calçados, com variadas alturas de saltos. Os principais resultados encontrados demonstraram que: $(I)$ independente da altura do salto houve maior ativação do GL, em comparação com o TA; (2) houve diferenças significativas do nível de ativação neuromuscular entre as situações de teste "pés descalços x salto alto" apenas para o músculo GL; e (3) quanto maior a altura do salto, maiores foram os percentuais de co-contração muscular encontrados. 
lunes e Santos (2005), ao avaliar a marcha de jovens, encontraram um aumento significativo da atividade muscular dos músculos GL e TA durante a utilização de calçados com salto alto, quando comparada à marcha com pés descalços. Esses resultados corroboram parcialmente com os achados do presente estudo, uma vez que não foi encontrado um aumento na ativação do tibial anterior, apenas no gastrocnêmio lateral, entre as três situações de teste.

Ainda, indo ao encontro dos achados do presente estudo, Santos et al. (2008) e Eisenhardt et al. (1996) relataram que, na marcha com salto alto, não ocorre o movimento de dorsiflexão devido à posição permanente da articulação do tornozelo em flexão plantar, e, considerando que quanto maior o salto do calçado, ocorre um maior deslocamento anterior do centro de gravidade, projetando o peso sobre o antepé, é possível aceitar que ocorra um maior recrutamento das fibras musculares do gastrocnêmio, o que justifica os maiores valores de ativação neuromuscular encontrados para este músculo no presente estudo. Do mesmo modo, Smith, Weiss e Lehmkuhl (1997) e Snow e Willians (1994) relataram que, durante a marcha, ao utilizar calçados de salto alto, os percentuais de distribuição do peso no pé se alteram, sendo que o peso sustentado pelo antepé aumenta de acordo com o aumento da altura do salto.

Ribeiro e Silva (2007), ao avaliar a marcha de jovens com salto alto, sugeriram que a articulação do joelho ficou limitada para exercer suas funções durante a marcha, possivelmente pelo fato desta articulação permanecer em um ângulo de flexão maior. O mesmo é demonstrado também por outros autores (OPILA et al., 1988; PHILLIPS et al., I991) e, ainda, tem sido relatado que, com a utilização de calçados de salto alto, há um aumento da freqüência de passos, redução do comprimento dos passos e redução da velocidade da marcha (SANTOS et al., 2008; EISENHARDT et al., 1996).

Conforme Ebbeling et al. (1994), o uso de calçados de salto, devido ao aumento da flexão plantar, gera um desequilíbrio corporal, pois resulta em uma diminuição da área de contato entre o pé e o solo, acarretando uma perda parcial da estabilidade, o que afeta o funcionamento de toda a cadeia cinética do membro inferior, principalmente da articulação do tornozelo. Deste modo, na marcha com calçado de salto alto, a estabilidade necessária para que esta marcha seja a mais natural e estável possível, resulta da co-contração do gastrocnêmio e tibial anterior. Além disto, tem sido demonstrado que, durante a marcha, a co-contração ocorre como um fator positivo para a estabilização dinâmica da articulação do tornozelo (FONSECA et al., 200 I; NUNES, 2004). Isto se deve à necessidade de maior estabilização, em ambos os membros inferiores, na fase de apoio. Este fenômeno parece ter ocorrido no presente estudo, na marcha com calçado de salto alto, a qual 
requer uma estabilização maior da articulação do tornozelo, resultando no aumento dos valores de co-contração com o uso do salto alto (Figura 3).

O uso freqüente e indiscriminado de calçados com salto alto por mulheres no seu dia-a-dia, por vezes por mais de 10 horas diárias, e em adolescentes e crianças em fase de desenvolvimento maturacional (IUNES et al., 2008) é preocupante, pois, além das características já descritas, a utilização diária deste tipo calçado pode resultar em fadiga dos membros inferiores e, de modo mais grave, no encurtamento dos músculos gastrocnêmio e sóleo, tornando-se, a longo prazo, um problema estrutural (GEFEN et al., 2002; KENDALL; McCREARY; PROVANCE, 1995). Tal fato pode restringir a flexão dorsal do tornozelo, ocasionar a hiperextensão do joelho na posição em pé (JAHSS, 1982), e resultar em desconforto às pessoas já habituadas ao uso do salto, no momento em que forem utilizar um calçado de sola plana (IUNES et al., 2008). Do mesmo modo, o uso freqüente de calçados de salto alto pode expor os usuários a transformações degenerativas da cartilagem articular, à ocorrência de osteoartrite (KERRIGAN; TODD; RILEY, I 998), e à ocorrência de dor na região metatarsiana (KENDALL; McCREARY; PROVANCE, 1995).

Assim, sabendo-se das implicações biomecânicas e cinesiológicas da utilização de calçados de salto alto, sugere-se, tanto para mulheres e/ou adolescentes, a utilização de calçados planos e/ou com menores alturas de salto. Jahss ( 1982), de forma mais pontual, sugere que, caso seja necessário a utilização diária de calçados de salto alto, estes saltos tenham, no máximo, 4 centímetros de altura.

Em suma, os resultados do presente estudo indicam que, na marcha com salto alto, existe uma maior ativação neuromuscular do gastrocnêmio lateral, possivelmente devido à quase inexistência de movimento na articulação do tornozelo, que permanece durante todas as fases da marcha em flexão plantar (SMITH; WEISS; LEHMKUHL, 1997). Assim, especula-se que a altura do salto parece estar associada diretamente com a ação do gastrocnêmio lateral, pois quanto mais alto for o salto, maior será a solicitação das fibras musculares, ou seja, maior é a ativação neuromuscular deste músculo. Entretanto, considerando o pequeno número amostral do presente estudo, entende-se que estes resultados são preliminares, necessitando de mais estudos, principalmente no que concerne ao percentual de co-contração durante a marcha de salto alto.

\section{CONCLUSÃO}

Os principais resultados encontrados no presente estudo demonstraram que: ( I) independente da altura do salto houve maior ativação do GL, em comparação com o TA; (2) o músculo GL apresentou maior ativação com o calçado de salto 
alto, em comparação à situação com os pés descalços; e (3) quanto maior a altura do salto, maiores percentuais de co-contração muscular foram encontrados.

\section{Activation and co-contraction of the gastrocnemius and anterior tibial muscles in the gait of women using different heights of heels}

ABSTRACT: The aim of this study was to investigate the muscle activation and co-contraction of tibialis anterior (TA) and lateral gastrocnemius (GL) during gait using different shoes. Nine college women walked on a treadmill with speed of $3,5 \mathrm{~km} / \mathrm{h}$ in three situations: without shoes, with short heels $(6 \mathrm{~cm})$, and high heels $(9 \mathrm{~cm})$ were simultaneously electromyography sinals recorded of TA and $G L$. The results showed that in the three test situations there was a higher activation of the $G L$ compared with TA $(p<0,05)$. There was also difference $(p<0,05)$ in the muscle activation between the conditions "without shoes $x$ high heels" just for the GL muscle. Finally, it was found that the higher the heel, higher is the percentage of co-contraction found. KEY-WORDS: Gait; electromyography; co-contraction; evaluation.

\section{Activación y co-contracción de los músculos tibial anterior y gastrocnemio en la marcha de las mujeres que utilizan las diferentes alturas de tacón}

RESUMEN: El objetivo de este estudio fue investigar la activación muscular y la co-contracción de los músculos tibial anterior (TA) y gastrocnemio lateral (GL) durante la marcha, utilizando zapatos diferentes. Nueve mujeres caminaron en una cinta de velocidad de $3,5 \mathrm{~km} / \mathrm{h}$ en tres situaciones: sin zapatos, con zapatos de tacón bajo $(6 \mathrm{~cm})$ y con zapatos de tacón alto (9 $\mathrm{cm}$ ), siendo al mismo tiempo registrado señales eletromiograficos de $T A$ y $G L$. Los resultados mostraron que en las tres situaciones de prueba había una mayor activación de $G L$ en comparación con TA $(p<0,05)$. También se encuentran diferencia $(p<0,05)$ en la activación muscular entre las condiciones sin zapatos x tacón alto sólo por el músculo GL. Por último, se encontró que, cuanto mayor es la altura del tacón, mayor sea el porcentaje de co-contracción. PALABRAS-CLAVES: Marcha; electromiografía; co-contracción; evaluación.

\section{REFERÊNCIAS}

AQUINO, C. F. et al. Mecanismos neuromusculares de controle da estabilidade articular. Revista Brasileira de Ciência e Movimento, Brasília, v. I2, n. 2, p. 35-42, jun. 2004.

CAMPOS, A. O. et al. Análise das alterações biomecânicas da força de reação do solo durante adaptação da marcha em esteira. Revista Brasileira de Biomecânica, São Paulo, v. 3, n. 5, p. 13-9, nov. 2002.

CANDOTTI, C.T.; LOSS, J.F. A produção científica brasileira na área de Biomecânica. Revista Brasileira Ciências do Esporte, Campinas, v. 28, n. I, p. 121 - 129, set. 2006. 
CARNAVAL, P. E. Cinesiologia aplicada aos esportes. Rio de Janeiro: Sprint, 2002

CHANG, K. K. et al. Forces patterns of heel strike and toe off on different heel heights in normal walking. Foot and Ankle International, Baltimore, n. 37, p. 22-28, june 2001.

CORRÊA, J. C. F. et al. Estudo eletromiográfico dos músculos vasto medial oblíquo e vasto lateral, no domínio temporal, durante a marcha. Revista Brasileira de Fisioterapia, São Carlos, v. 6, n. I, p. 37-40, jan./abr. 2002.

EBBELING, C. J. et al. Lower extremity mechanics and energy cost of walking in high-heeled shoes. Journal of Orthopaedic and Sports Physical Therapy, Alexandria, v. 19, n. 4, p. I90-196, apr. 1994.

EISENHARDT, J. R. et al. Changes in temporal gait characteristics and pressure distribution for barefeet versus various heel height. Gait \& Posture, Oxford, v. 4, n. 4, p. 280-286, 1996.

FONSECA, S. T. et al. Análise de um método eletromiográfico para quantificação de co-contração muscular. Revista Brasileira Ciência e Movimento, Brasília, v. 9 n. 3, p. 23-30, jul. 200 I .

GEFEN, A. et al. Análisis of muscular fatigue and foot stability during high-heeled gait. Gait \& Posture, Oxford, v. I 5, n. I, p. 56-63, feb. 2002.

HAMILL, J.; KNUTZEN, K. Bases biomecânicas do movimento humano. São Paulo: Manole, 1999.

IUNES, D. H.; SANTOS, C. Análise eletromiográfica da atividade muscular durante a marcha em crianças utilizando diferentes tipos de calçados. Fisioterapia Brasil, Rio de Janeiro, v. 6, n. 5, p. 328-331, set./out. 2005.

IUNES, D. H. et al. A influência postural do salto alto em mulheres adultas: análise por biofotogrametria computadorizada. Revista Brasileira de Fisioterapia, São Carlos, v. I2, n. 6, p. 44I-446, nov./dez. 2008.

JAHSS, M. H. Disorders of the foot \& ankle: medical and surgical management. Philadelphia: WB Saunders, 1982.

KENDALL, F. P.; McCREARY, E. K.; PROVANCE, P. G. Músculos: provas e funções. São Paulo: Manole, 1995.

KERRIGAN, D. C.; TODD, M. K.; RILEY, P. O. Knee osteoarthritis and high heeled shoes. Lancet, London, v. 5I, p. 399-40I, may 1998

KONRAD, P. The ABC of EMG: a practical introduction to kinesiological electromyography. New York: Noraxon, 2005.

MCGINNIS, P. M. Biomecânica do esporte e exercício. Porto Alegre: Artmed, 2002

NUNES, L. C. B. G. Efeitos da eletroestimulação neuromuscular no músculo tibial anterior de pacientes hemiparéticos espásticos. 2004. 105 f. Dissertação (Mestrado em Engenharia 
Elétrica) - Faculdade de Engenharia Elétrica e de Computação, Universidade Estadual de Campinas, Campinas, 2004.

OPILA, K. A. et al. Postural aligment in barefoot and high-heeled stance. Spine, Philadelphia, v. 13, n. 5, p. 542-547, may 1988.

PHILLIPS, R. D. et al. Modification of high heel shoes to decrease pronation during gait. Journal of the American Podiatric Medical Association, Washington, v. 81, n. 4, p. 21 5-219, apr. 1991. RIBEIRO, F.; SILVA, J. Estudo comparativo da marcha de crianças com e sem o uso de calçado de salto. In: CONGRESSO BRASILEIRO DE BIOMECÂNICA, 12., Estância de São Pedro, 2007. Anais... Estância de São Pedro: UNESP, 2007.

SANTOS, C. L. et al. Repercurssões biomecânicas do uso de salto alto na cinemática da marcha: um estudo retrospectivo de 1990 a 2007. Revista Educação Física, Rio de Janeiro, n. 143 , p. 47-53, dez. 2008.

SENIAM. Surface ElectroMyoGraphy for the Non-invasive Assessment of Muscles. SENIAM project: 2008. Disponível em: www.seniam.org.

SILVA, J. Análise eletromiográfica dos músculos sóleo e gastrocnêmio de mulheres com uso de calçado de salto. In: CONGRESSO BRASILEIRO DE BIOMECÂNICA, I2., Estância de São Pedro, 2007. Anais... Estância de São Pedro: UNESP, 2007.

SMITH, L. K.; WEISS, E. L.; LEHMKUHL, L. D. Cinesiologia clínica de Brunnstrom. São Paulo: Manole, 1997.

SNOW, R. E.; WILLIAMS, K. R. High heeled shoes: their effect on center of mass position, tree-dimensional kinematics, rearfoot motion and ground reaction forces. Archives of Physical Medicine and Rehabilitation, Chicago, v.75, n. 5, p. 568-576, may 1994.

WINTER, D. A. Biomechanics and motor control of human movement. New York: Wiley, 2005.

Recebido: 03 ago. 2010

Aprovado: 12 jun. 2011

Endereço para correspondência:

Cláudia Tarragô Candotti

Programa de Pós-Graduação em Ciências do Movimento Humano Escola de Educação Física da Universidade Federal do Rio Grande Do Sul Rua Felizardo 750, Jardim Botânico - Porto Alegre/RS CEP: $90690-200$ 